\title{
Embera Wera: vida, poder y resistencia ${ }^{1}$
}

\section{Embera Wera: Life, power and endurance}

\author{
Embera Wera: a vida, \\ poderes e resistência
}

\author{
John Edison Sabogal Venegas ${ }^{2}$ \\ joesabogalve@unal.edu.co \\ Universidad Nacional de Colombia \\ Bogotá - Colombia
}

Artículo recibido: 14/04/2014

Artículo aprobado: 18/06/2014

Para citar este artículo: Sabogal, J. (2014).

Embera Wera: vida, poder y resistencia.

Ciudad Paz-Ando, 7(1), 198-214

DOI: http://dx.doi.org/10.14483/udistrital.jour. cpaz.2014.1.a11

\footnotetext{
1 Buena parte de lo consignado en este texto corresponde a los resultados del proceso investigativo realizado en 2013 (Sabogal, 2014).

2 Psicólogo de la Universidad Nacional de Colombia. Antropólogo en formación de la misma institución.
}

\section{Resumen}

El presente artículo busca proponer algunas reflexiones sobre la interacción entre violencia, relaciones de poder y dinámicas de resistencia a partir de los relatos de vida de una mujer indígena Embera en condición de desplazamiento en Bogotá. Reconstruyendo sus experiencias personales e identificando los hitos más importantes de la vida de esta mujer, se establecen las paradojas, conflictos y formas de violencia que las mujeres indígenas experimentan y que se encuentran representados en parte por los relatos personales recopilados. Partiendo de la indeleble relación entre poderes y resistencias, al final del texto se proponen algunas reflexiones sobre las fugas, transgresiones y fronteras identitarias de mujeres indígenas en contextos urbanos.

Palabras clave: Embera, indígenas, violencia, poder, resistencia. 


\begin{abstract}
The aim of this paper is to suggest some reflections on interactions between violence, power relationships and resistance dynamics from the life stories of an Embera woman on displacement conditions in Bogotá. By reconstructing her personal experiences and indentifying the most important landmarks in this woman's life, this article sets paradoxes, conflicts and forms of violence to which indigenous women are exposed and which are represented, partly, by the personal stories gathered in here. From the indelible relationship between powers and resistances, this paper proposes some reflections on escapes, transgressions and identity borders of indigenous women in urban contexts.
\end{abstract}

Keywords: Embera, indigenous, violence, power, resistance.

\section{Introducción}

a vida es inaprensible en su entera complejidad, sus claroscuros y caóticos momentos requieren a veces un trato entre lo académico y lo literario, si bien no para abarcarla completamente, sí para desentrañar algunos de sus acordes y disonancias. Pareciera que hay que resignarnos, en el mejor de los casos, a trabajar sobre pisos movedizos e intentar bosquejar siluetas personales, perpetuando un sacro temor a los misterios vitales. A lo largo del artículo pretendo reflexionar -a partir de los relatos de vida de Jimbûso Wera $^{3}$, una mujer indígena

3 El nombre verdadero ha sido cambiado por un nombre ficticio en Embera que significa Mujer Colibrí, con el fin no solo de proteger su identidad y privacidad, sino de acentuar en su historia el microcosmos Embera que representa sin descuidar a su vez las particularidades de sus experiencias vitales, que reflejan, como el colibrí, una movilidad incesante.

\section{Resumo}

O objetivo deste artigo é sugerir algumas reflexões sobre as interações entre a violência, relações de poder e dinâmicas de resistência das histórias de vida de uma mulher, Embera que vive em condições de deslocamento em Bogotá. Ao reconstruir suas experiências pessoais e identificar os marcos mais importantes na vida desta mulher, este artigo define os paradoxos, os conflitos e as formas de violência a que as mulheres indígenas estão expostos e que são representados, em parte, pelas histórias pessoais recolhidos aqui. Da relação indelével entre poderes e resistências, este trabalho propõe algumas reflexões sobre fugas, transgressões e as fronteiras da identidade das mulheres indígenas em contextos urbanos.

Palavras-chave: Embera, indígenas, violência, poder, resistência.

Embera en condición de desplazamiento en Bogotá- las complejas relaciones entre experiencias vitales, relaciones de poder y dinámicas de resistencia, enfatizando en particular las paradojas y conflictos de las mujeres indígenas en las ciudades. Una buena parte de lo consignado en el presente texto se fundamenta en el proceso investigativo realizado en el 2013 como trabajo de grado para optar por el título de psicólogo (Sabogal, 2014). A pesar de este hecho, no busco presentar una lectura psicologista o puramente disciplinar de las dinámicas investigadas, por lo cual recurro a diferentes teorías y perspectivas. De forma reflexiva, existe por supuesto un locus de enunciación particular que restringe las posibilidades del artículo: un hombre no indígena de ciudad que habla sobre la vida de 
una mujer indígena en condición de desplazamiento en la urbe. Esta condición implica que lo aquí presentado dista completamente de ser la última palabra, es, por el contrario, una reconstrucción biográfica a dos voces que busca problematizar las intersecciones entre vida, poder y resistencia.

Con el fin de contextualizar las experiencias biográficas de Jimbûso Wera, parto de una breve caracterización social y cultural del pueblo Embera, para posteriormente centrarme en la reconstrucción y análisis de los relatos sobre diferentes experiencias de Jimbûso Wera en su tránsito de lo indígenatradicional a lo urbano-occidental. El apoyo en los relatos de vida como parte del conjunto de métodos biográficos existentes es una de las formas de acercamiento a sujetos históricamente excluidos (Archila, 1998), toda vez que permite escuchar voces subalternizadas, tanto por la sociedad, como por la academia. Para finalizar, sostengo algunas reflexiones sobre los intersticios entre vida, poder y resistencia como una forma particular de comprender las problemáticas y paradojas de los indígenas -particularmente las mujeres- a raíz de diversas formas de violencia y conflicto que vive el país.

\section{La gente del maíz}

Embera en español significa "gente" o "humanidad" (Losonczy, 2006). Los antiguos Embera eran Bembera: "Gente del maíz" (Vasco, 2002), pobladores milenarios del Pacífico colombiano y panameño. En la actualidad, los Embera son uno de los pueblos indígenas más grandes del país con aproximadamente 93.000 personas $^{4}$, que aunque están organi-

4 Cantidad aproximada a partir de los datos que el Ministerio de Cultura de Colombia utiliza según las cifras oficiales emitidas desde el Censo realizado en el 2005 por el Departamento Administrativo zados en grupos relativamente diferenciados, comparten similitudes sociales, culturales e históricas que los identifican como nación indígena. A nivel histórico, hacia el final del siglo XIX y principios del XX, la constitución de nuevos poblados hacia el interior del país en las zonas más montañosas llevaron a la desintegración de resguardos Embera y facilitaron los procesos de colonización en los tiempos de la república (Ulloa, 1992). La mitad del siglo XX marca el comienzo de la llegada de población blanca a los territorios habitados por los Embera, en su mayoría campesinos pobres procedentes de Antioquia y Caldas que buscaban tierras para trabajar; aunque posteriormente serán grandes terratenientes y latifundistas los que ocuparán territorios habitados por los Embera, instaurando relaciones asimétricas y jerárquicas con las comunidades (Vasco, 1975).

Actualmente, los Embera viven principalmente en asentamientos dispersos ubicados en el occidente colombiano, aunque por su histórica tendencia a la migración y dispersión (Vasco, 1975; 1990), se encuentran en diversos departamentos del país como Chocó, Risaralda, Antioquia, Valle del Cauca, Caldas, Córdoba, Putumayo y Caquetá, entre otros (Losonczy, 2006). Su lengua, el Embera, hace parte de la familia lingüística Chocó emparentada con el idioma Wounan (Ulloa, 1992). Entre los Embera se distinguen los Dóbida, o "gente de río", quienes viven en las riveras de afluentes hídricos; los Oibida, o habitantes de la selva; y los Eyadiba, u hombres de montaña (Losonczy, 2006; Ministerio de Cultura, 2010a). A partir de una división más generalizada actualmente, los Embera se dividen en cuatro grupos con diferencias dialectales, culturales y territoriales relativamente identifi-

Nacional de Estadística (DANE). 


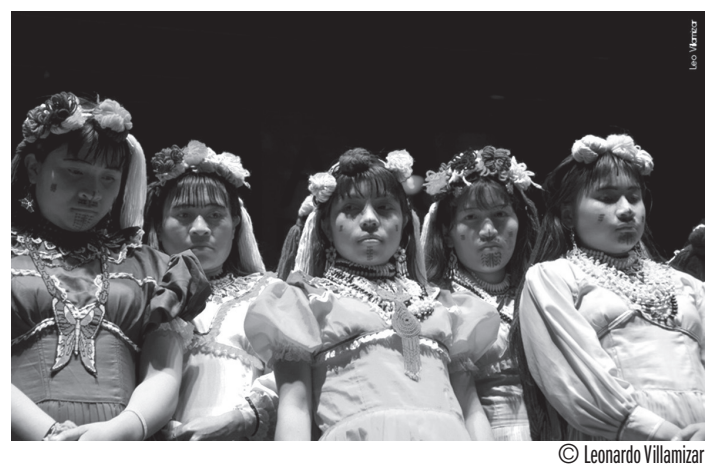

cables: Embera Chamí, ubicados en regiones montañosas (p.e eje cafetero); Embera Katío, distribuidos en el Chocó y en algunas zonas altas de la geografía nacional; los Dóbida y los Eperara Siapidara, estos dos últimos con una menor población y respectivamente residentes del norte del Chocó y del sur del litoral Pacífico en los departamentos de Cauca y Nariño (Ministerio de Cultura, 2010b).

Los Embera ${ }^{5}$ se caracterizan por ser grupos especialmente agricultores, que practican otras actividades para la manutención como la caza, la pesca y la recolección (Vasco, 1975). Los principales alimentos cultivados son el maíz, el plátano y el chontaduro, dependiendo la zona; y en menor proporción, el café, el arroz y la caña, entre otros, complementan sus cultivos (Ulloa, 1992). Los Embera son un pueblo eminentemente endogámico (Vasco, 1975; Losonczy, 2006), organizado generalmente en familias nucleares, de carácter patrilineal y patrilocal, es decir, que tanto la filiación ascendente como descendente y la residencia postmarital se realizan a partir del varón, aspecto que puede dar luces del papel de la mujer Embera. Su organización política tradicional no cuenta con una jefatura permanente ni centralizada (Losonczy, 2006),

5 Aunque la caracterización socio-cultural siguiente recopila literatura etnológica sobre los Chamí, principalmente, por ser la comunidad a la que pertenece Jimbûso Wera, tales descripciones son en su mayoría rasgos generalizables a los otros grupos Embera. aunque en la actualidad los resguardos cuenten con cabildos y gobernadores, los cuales han conformado organizaciones políticas importantes como la Asociación de Cabildos Indígenas del Chocó (OREWA) y la Organización Indígena de Antioquia (OIA). Una figura central en su organización social es el jaibaná, o médico tradicional, quien es el encargado de curar enfermedades y mediar entre los Embera y los jais, que pueden entenderse como espíritus o esencias de todas las entidades existentes (Vasco, 1975; 1990). Dentro de la cultura material Embera cabe destacar la cestería (Ulloa, 1992), realizada principalmente por las mujeres utilizando bejucos naturales, la pintura corporal -que está cada vez más en desuso- y el tejido en mostacilla que ha jugado un papel importante como salida económica para las familias desplazadas hacia ciudades o cabeceras municipales (Cabrera, et al., 2009). Igualmente importante, es la tradicional y marcada división de tareas entre hombres y mujeres que existe en el pueblo Embera en la cual los primeros se encargan de la agricultura y de educar a los niños, mientras las mujeres realizan las tareas del hogar y enseñan a las niñas lo pertinente para convertirse en Embera Wera, es decir, en mujeres Embera.

Actualmente, una de las problemáticas más complejas del pueblo Embera es el desplazamiento forzado de sus territorios de origen, puesto que más allá de su histórica segmentación y dispersión (Vasco, 1975), las problemáticas a consecuencia del olvido del Estado y las dinámicas del conflicto armado han llevado a los Embera a migrar hacia cabeceras municipales y ciudades como Medellín, Pereira, Cali y Bogotá. Según Cabrera, et al (2009), en los procesos de migración y desplazamiento del pueblo Embera se pueden identificar históricamente tres "olas" o mo- 
mentos. La primera comienza en la década de los 70's con los progresivos descubrimientos de minas de oro y el respectivo proceso colonizador por parte de comerciantes y mineros. La segunda ola tuvo lugar entre los 80's y los 90's con la incursión de grupos armados (primero el M-19 y luego el ELN, las FARC y grupos paramilitares), que comenzaron a disputar el dominio del litoral Pacífico y ciertas zonas cafeteras. Por último, la tercera ola de desplazamientos ocurrió a principios del siglo XXI con el recrudecimiento de enfrentamientos, intimidaciones, extorsiones y demás acciones violentas contra las comunidades indígenas, que los llevaron a migrar y buscar protección en cabeceras municipales y ciudades. Por estas razones, el desplazamiento actual de los Embera hacia las ciudades está ligado -como gran parte de las dinámicas del conflicto armado- a disputas por la tierra con orígenes históricos, que los ha llevado a constantes desplazamientos a nuevos territorios, lo que ha conducido al inevitable agotamiento de estas áreas de salvaguarda Embera y a la búsqueda de la ciudad como lugar de protección.

\section{Vida en movimiento: Jimbûso Wera}

A nivel general, Latinoamérica vive un paulatino pero cada vez más fuerte proceso de etnización de sus contextos urbanos (Yanes, 2004). Por disímiles motivos y dinámicas, los pueblos originarios han arribado a la ciudad, generando la transformación tanto de prácticas tradicionales indígenas como de dinámicas políticas, económicas y sociales de grandes centros urbanos. Los estudios y reflexiones académicas han abordado este proceso desde diferentes perspectivas, enfatizando distintas situaciones y dinámi- nes con los Mapuches en Chile y Argentina (Merino \& Tileagâ, 2011; Briones, 2010). Sin embargo, las migraciones de mujeres indígenas a las ciudades ha sido una temática generalmente subvalorada (Oehmichen, 1999), situación a la cual no escapan los trabajos investigativos realizados con los Embera en ciudades (Cabrera et al., 2009; Gaitán, 2010 y Mejía, 2007). Las múltiples formas de violencia y exclusión (económica, social, política, simbólica, etc.) que padecen los pueblos indígenas, que se sustentan en lógicas de colonialidad y subalternización de lo no "moderno-occidental” (Castro-Gómez, 2005), se agudizan para las mujeres indígenas como las Embera, quienes representan en el imaginario urbano lo menos moderno o civilizado, pues -retomando la expresión de Marisol de la Cadena (1991)- se cree que "las mujeres son más indias". Dentro de este marco, me centraré a continuación en la reconstrucción biográfica de Jimbûso Wera, para lo cual he identificado analíticamente varios momentos o hitos vitales que conllevan muchas veces a paradójicas dinámicas y relaciones entre lo indígena y lo no indígena.

El primer momento vital es el proceso de iniciación al mundo Embera, en el cual Jimbûso Wera se narra a partir de los aprendizajes fundamentales de la familia y la comunidad, en donde son las diferencias de género las que marcan principalmente los contenidos y las dinámicas de su proceso de construcción como sujeto. El segundo hito es la irrupción de los procesos de educación propia producto de su ingreso a un internado de religiosas en territorio, marcado por dinámicas de colonialidad y subalternización de lo indígena. El comienzo de la vida adulta y la conformación de la familia es el tercer momento experiencial. Posteriormente, como cuarto hito se identifica la salida del territo- 
rio a raíz del conflicto armado y las tensiones entre la ciudad imaginada y la vivida, con la respectiva exigencia de nuevas formas de ser y conocer en el contexto urbano. El quinto momento hace referencia a las dinámicas de agencia, particularmente marcado por la separación con su esposo, en la cual se identifica con fuerza cómo Jimbûso Wera resiste a ciertas relaciones de dominación, lo cual no impide apreciar las contradicciones y los cambios que experimenta su vida en la ciudad. En el último momento se identifican el liderazgo comunitario en la ciudad así como sus experiencias educativas en espacios de educación formal en Bogotá, que han construido a Jimbûso Wera como una mujer indígena en la frontera entre lo tradicional y lo urbano-occidental.

A lo largo de estos momentos, apuntaré ciertas interpretaciones que tienen como eje la intersección entre las relaciones de poder, las formas de violencia y las dinámicas de resistencia. Estas interpretaciones se mueven a partir de consideraciones teóricas que las fundamentan, en particular, la importancia de observar las relaciones humanas como constituidas por el ejercicio del poder (Foucault, 1992), por una microfísica particular en la cual no sólo operan dimensiones opresoras o restrictivas, sino lógicas de poder que crean y producen sujetos e interacciones. Partiendo de Foucault (1992), dos consideraciones importantes emergen: en primer lugar, el poder no se posee sino que se ejerce, se distribuye de forma dispersa y móvil en cada relación social; de igual forma, como segunda afirmación, todo poder conlleva un ejercicio de resistencia, una contraposición constituyente. Retomando a Scott (citado en Peñaranda, 2012, p.14) la resistencia puede ser vista como "cualquier acto de un grupo subordinado, dirigido a atenuar las imposiciones de un grupo dominante o, también, a hacer avanzar sus demandas propias". La importancia de analizar a lo largo de la vida de Jimbûso Wera poderes y resistencias, no descuida, sin embargo, los distintos modos en que la(s) violencia(s) se expresa(n), sus dimensiones económicas, sociales, políticas y subjetivas, que marcan la vida de las mujeres indígenas en contextos urbanos.

Jimbûso Wera nace el 13 de octubre de 1980 en Mistrató, Risaralda. Sus primeros años de vida estuvieron marcados por un conjunto de experiencias centradas en lo que he denominado su iniciación al mundo cultural de la gente del maíz. Para Jimbûso Wera, y en general para las mujeres de la comunidad, la madre juega el rol de educadora principal. Como lo menciona el padre de Jimbûso Wera: "A las mujeres les hacen aprender [...] las mamás. [...]. Sí, y los hombres le hacen aprender los padres, los papás ${ }^{\prime \prime}$. Esta separación fuertemente marcada conlleva una iniciación educativa "generizada", una división del conocimiento a través del género, en donde las mujeres aprenden las labores del hogar (cocinar, lavar y cuidar los niños), propias del ámbito social Embera privado, mientras los hombres aprenden trabajos exteriores como la caza, la pesca y la agricultura. Según Jimbûso Wera:

Allá indicaba solo hacer [...] culturas que nosotros manejamos $\downarrow$, [...]enseñan a nosotros como para aprender hacer de comer $\uparrow$, la mamá [...] porque dicen que uno nunca se va a vivir solo, siempre va a tener su pareja, entonces para poder cuidar bien entonces le enseñan a uno desde::, cuando ya esté señorita ya le mandaban a hacer, cuidar a los hermanitos $\uparrow$, eh:: bueno, hacer de todo, como de todo, loza,

6 Las diferentes convenciones de transcripción son tomadas y adaptadas de Potter (1998, pp.293-294) con el fin de no perder completamente la riqueza de la oralidad en el texto. 
de todo. [...], yo tengo cinco hermanitos, entonces yo cuidaba a ellos mientras que mi mamá se iba a otro lugar $\downarrow$.

Estos procesos de educación propia y tradicional encuentran su escenario fundamental en la familia (Vasco, 1975); sin embargo, no se desligan de relaciones comunitarias más amplias, especialmente en el caso de los hombres, quienes exploran bajo la tutoría del padre la exterioridad del hogar. En este primer momento vital, la vida de Jimbûso Wera no se limita como pareciera a la transmisión de conocimientos básicos para la reproducción material de la comunidad, van más allá de asegurar el establecimiento de nuevos núcleos familiares, fundamentándose en la reproducción y trasmisión simbólica de la cultura Embera. En una fusión cotidiana, la dimensión laboral-material y la simbólico-cultural, se imbrican con el fin de iniciar a niños y niñas en la vida Embera, por lo cual las mujeres aprenden, por ejemplo, a tejer como actividad fundamental de su aprendizaje:

La época cuando yo crecí [...] en resguardo::, nosotros, pues manejábamos $\uparrow$ toda la cultura, desde la mamá pues que enseñan a trabajar a unot. Nosotros siempre tejemos de esos bejucos [...] Todos esos tejidos, como bejucos, mi mamá me enseñó como cuando yo tenía de cin-, siete años adelante yo sabía tejer de esos.

Como un quiebre en las experiencias que vivía en el marco de los procesos de educación propia, Jimbûso Wera estudió en un internado de monjas de la Madre Laura que había llegado a territorio Embera hacia 1933 (Vasco, 2002). Este nuevo escenario de educación formal constituye un proceso de ruptura/apertura experiencial en el marco de un proceso de enseñanza-aprendizaje institucionalizada y estructurada desde cánones occidentales, el cual tiene como intención primordial evangelizar e imponer esquemas de creencia occidental. Este objetivo final se basa en el ejercicio de la violencia simbólica y la colonialidad, entendida como lógica de subalternización y de imposición de esquemas occidentales de ser y pensar (Castro-Gómez, 2005). Es fundamental señalar que el padre de Jimbûso Wera había estudiado igualmente en el internado, padeciendo no solo atropellos culturales como la prohibición de hablar Embera, sino maltratos físicos (Sabogal, 2014); sin embargo, él mismo decide enviar a su hija Jimbûso Wera cuando tenía ocho años al internado. Aunque en la época en la cual su padre estudió con las misioneras había resistencia por parte de la comunidad de enviar a estudiar a las mujeres, por considerar que conllevaba vicios como la prostitución y la pérdida de la identidad cultural, según lo relata Jimbûso Wera, las monjas "fueron diciendo [...] «a las niñas también tienen que dejar aprender porque ellas igual son::, ellas son, igual son humanos y tiene pensamiento igual que los hombres» [...] entonces ya dejaron estudiar a las mujeres. Paradójicamente, esta transformación aparentemente democrática de las relaciones de género, agudizó los procesos de colonialidad y despersonalización cultural al introducir tanto a niños como a niñas a la cultura occidental y limitar su socialización en el marco de la cultura Embera tradicional (Vasco, 1975). En un plano hermenéutico, la efectividad de estos mecanismos de poder se evidencia en el hecho de que son los mismos Embera quienes comienzan a legitimarlos. Este paradójico hecho, en el cual quien es excluido acepta y legitima la reproducción de las condiciones de exclusión, no sólo emerge por la coacción sino por la fuerza de la violencia simbólica, es decir, la imposición de significa- 
dos y el ejercicio de violencia ejercido sobre un sujeto con su consentimiento (Bourdieu \& Wacquant, 2005). Esto quiere decir que las relaciones de poder/subalternización de hombres y mujeres Embera operan fundamentalmente desde una dominación simbólica que "implica una forma de complicidad que no es ni sumisión pasiva a una coerción exterior, ni adhesión libre a valores [...] por parte de quien la sufre, implica una actitud que desafía la alternativa corriente, de libertad-coacción" (Bourdieu \& Wacquant, 2005, p.240).

Sin embargo, en la lectura subjetiva que realiza Jimbûso Wera de esta experiencia, ella considera importante ciertos aspectos de su paso por el internado, en particular el aprendizaje del español y, por paradójico que parezca, las posibilidades que esta educación le brindó para ser más autónoma en relación con los hombres:

en la época eso era muy difícil aprender español, yo no sabía hablar nada y las monjitas nos enseñaron $\uparrow$. [...] las monjitas nos enseñ[aron] hablar el español. Pues si no fuera eso, nosotros no íbamos a saber hablar nada $\uparrow[. .$.$] . Mi$ papá dice que siempre tenía pensamiento que yo tenía que salir adelante $\downarrow$, siempre 0 sea para que yo no sea así, hombres que:.. Lo que pasa es que allá es costumbre^ que los hombres se manipulan a las mujeres.

Al ser el español la lengua de comunicación entre indígenas y no indígenas, su aprendizaje y buen uso -que se presenta con mayor frecuencia en hombres que en mujeres- permite una apertura experiencial gestada paradójicamente en la ruptura con la tradición que representa el internado. Este aspecto será fundamental en su llegada a Bogotá, pues le abrirá otras posibilidades y le permitirá acceder a espacios importantes en la ciudad, una diná- mica de resistencia y uso contra-hegemónico de mecanismos de coacción como la lengua. Como lo sostiene Ulloa, las mujeres indígenas "han tenido que hacer frente a procesos de exclusión en contextos urbanos y ganar espacios de participación en escenarios académicos, laborales e institucionales" (2007, p.20), los cuales se reflejarán en la vida de Jimbûso Wera al llegar a Bogotá.

Para los Embera no existe una etapa definida entre la infancia y la adultez (Vasco, 1975), la pubertad o la adolescencia no son un periodo culturalmente marcado, por lo cual los niños y niñas van participando y aprendiendo en la práctica las actividades propias de la vida adulta, insertándose de forma gradual -a pesar de la ruptura que representa el internado- en la adultez. Puesto que contraen matrimonio (católico) entre los 14 y 16 años, los hombres deben conocer el trabajo agricultor mientras que las mujeres deben haber aprendido a realizar las labores del hogar. En el caso de Jimbûso Wera, el tránsito hacia la vida adulta ocurre también de una forma relativamente abrupta, pero tiene como escenario la ciudad, puesto que después de estudiar cerca de cinco años en el internado, ante una recaída de salud de su madre, viajan con su padre a Pereira, una ciudad que funciona como un lugar de paso, un punto intermedio entre el territorio tradicional y el espacio urbano en toda su dimensión como lo puede ser Bogotá. En Pereira, Jimbûso Wera comienza a vivir con una tía y deja definitivamente de estudiar, estableciendo a los 14 años una relación de pareja con un hombre Embera: "Nos vinimos a Pereira porque mi mamá se puso muy mal de salud y, ahí [...] yo encontré un muchacho y me fui a vivir con él. Ya dejé de estudiar, no pude estudiar^". Esta decisión, que implicó el tránsito hacia un nuevo camino vital al convertirse en esposa y madre, no 
tuvo el respaldo de sus padres, lo cual conllevó incluso a la ruptura de su relación por un tiempo. Después de dos años de no hablar con ellos, recuperaron el contacto bajo la exigencia del padre de casarse por la iglesia: "Mi papá dijo que [me] casara por la iglesia porque ellos son casados por la iglesia, son católicosł", suceso que evidencia nuevamente ejercicios de colonialidad e imposición de lo occidental legitimados y reproducidos por los mismos Embera.

En el marco de las nuevas experiencias que vivía en esta ciudad intermedia, Jimbûso Wera entró en el mundo del trabajo, cuidando niños y laborando en una casa de familia: "en esa época a mí me gustaba también ayudar a mi marido, mi marido trabajaba y yo también^, me gustaba trabajar para ayudar ambos". Tuvo cuatro hijos y, posteriormente, después de vivir algunos años en Pereira, decidió junto con su esposo volver a territorio indígena y vivir en la finca de sus suegros. En este nuevo espacio se aprecia con mayor claridad su papel como educadora de sus hijos, especialmente con su hija mayor a quien educó en las mismas labores que su madre le había enseñado cuando pequeña:

Yo ya allá [en territorio], traje la niña grandecita tenía nueve añitos $\uparrow$. Yo le enseñé también a la lavar ropa, a hacer de comer mientras la niña tenía diez añitos ella ya sabía preparar almuerzo $\uparrow$, ya sabía cuidar sus hermanitos, todo esoł. Y pues ella cuando ya se casó no tuvo ningún problema de que no sabía lavar ni ropa ni nada, o sea, en esa parte sí enseñamos desde muy niñas. Ella ya sabía tejer chaquira también [...] canastos [...] con bejucos.

Este hecho evidencia que el territorio, más que ser un contexto adyacente de la transmisión de saberes tradicionales, es el espacio social

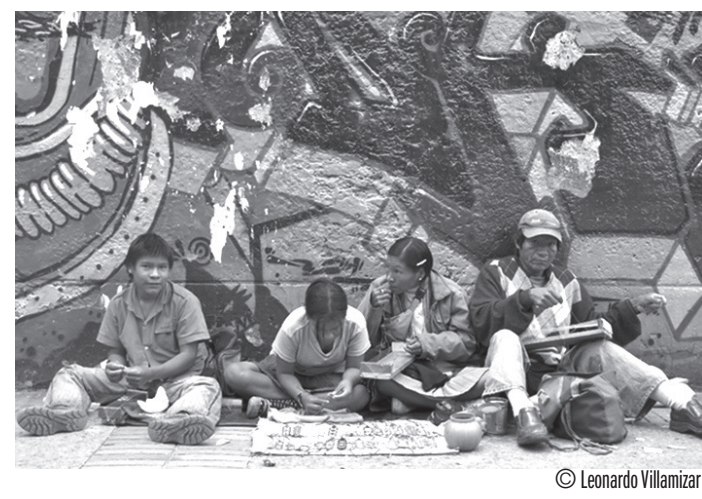

por excelencia de la reproducción cultural Embera, pues representa el lugar donde se construye un nosotros relativamente diferenciado y alejado del otro occidental, por lo cual no es exagerado decir que los territorios indígenas se configuran muchas veces como espacios culturales de resistencia, como lo refleja el paradigmático ejemplo del Cauca (Peñaranda, 2012). Un evento fundamental, que origina el desplazamiento por parte de Jimbûso Wera y el resto de su familia, es la agudización de las dinámicas del conflicto armado a mediados de la década de los noventa, caracterizada por la consolidación de la hegemonía de la guerrilla en la zona y los enfrentamientos con las fuerzas armadas. Este nuevo actor ejercía su poder por medio de las armas, acompañando la violencia física y bélica con violencia simbólica, limitando la autonomía indígena y coaccionando a la comunidad. Mientras que el dominio de los misioneros se basaba en la fuerza de su imposición cultural, la hegemonía de los grupos armados tiene su asidero en el poder bélico, como lo relata el padre de Jimbûso Wera:

[... el ellos nos vinieron conquistando, con el cabildo mayor y tanto los gobernadores $\uparrow$ y tanto la comunidad $\downarrow$. Entonces ellos nos decían que ellos son ejércitos del pobre, [...] nos orientaban† que «porque los indígenas hoy no tienen armas que para enfrentar con el gobierno» que ellos 
van a responder, [...] cuando hay problemas de, de territorio [...] como tienen sus armas que ellos iban a favorecer con esas armas.

Una de las dinámicas más complicadas fue la relación misma entre los grupos armados y la figura del jaibaná, puesto que además de desestabilizar el papel mediador del médico tradicional con los jais, su asesinato implicaba el debilitamiento de las creencias, los saberes y los poderes tradicionales de los Embera. Por esta razón, no es exagerado argumentar que la violencia socio-política que vive el país, no sólo pasa por encima de vidas y comunidades, sino que arrasa tradiciones milenarias, sabedores y prácticas culturales indígenas ( $\mathrm{Vi}$ Ila \& Houghton, 2005). Para esta época, Jimbûso Wera había decido vivir un tiempo con sus padres pues su compañero sentimental la maltrataba y había intentado en repetidas ocasiones separarse, aspecto que analizaremos más adelante. Finalmente, tras el agudo conflicto en la zona y las amenazas directas hacia el padre de Jimbûso Wera, éste decide salir junto con su esposa hacia Pereira con el fin de proteger sus vidas, suceso que es narrado por ella de la siguiente forma: "yo me fui a vivir con mi papáł. Después que mis papás salieron ya me quedé sola porque todos no quedaron, o sea mi papás, mis hermanos todos salieron, entonces yo me quedé solał. Yo me quedé aburrida". Tiempo después, Jimbûso Wera, quien para la época tenía 28 años, decidió salir junto a su esposo e hijos hacia Bogotá, puesto que sentían que estaban en peligro y veían a esta ciudad como un lugar de nuevas oportunidades:

Ahí llegaban guerrilleros despuesito, nosotros no aguantamos venimos porque de pronto nos podían matar [...] Yo viví como dos meses después de que salieron mis papás. Después me salí y ya jamás me volvíł. Con mi esposo y mis hijos todos nos salimos.

Una vez llegada a Bogotá, la implacable fuerza de la urbe se hacía sentir en la historia vital de Jimbûso Wera: "No, yo pensé que era bueno, así como yo vivía en Pereira, yo ya acostumbrada pues a vivir en la ciudad. Pero aquí sí fue terrible, le cuento". El reto que se presentaba era entrar a la lógica urbana, caótica, azarosa y saturada de interacciones, una forma de relación superficial que era diametralmente opuesta a la dinámica comunal vivida en el territorio. Las demandas económicas que se imponían fueron uno de los primeros retos con los cuales Jimbûso Wera se enfrentó. Los inminentes constreñimientos económicos le eran complejos de afrontar, al punto de tomar decisiones difíciles como vender su cabello: "me pasó muchas cosas, hasta me tocó mandarme a mochar mi cabello^, porque yo tenía un cabello muy largo y me compraron porque tuve que vender para poder pagar arriendo". Esta serie de demandas económicas implicaban la necesidad de buscar labores que permitieran la satisfacción de las necesidades básicas y la estabilización económica en la ciudad. Otras familias Embera desplazadas con anterioridad habían encontrado en el ejercicio de la mendicidad un medio de supervivencia en Bogotá; sin embargo, desde su llegada a la ciudad, Jimbûso Wera se resistió a esta opción, puesto que la consideraba una actividad indigna y fuente de vergüenza:

[... a aquí como la mayoría que llegaba siempre ellos eran:..., 0 sea pedían muchas colaboraciones en la calle $\uparrow$, pedía moneda y todo, pero yo como uno no es acostumbrado de eso, uno le da mucha pena, entonces yo nunca, más bien no voy a pedir así, me da pena, «a uno no le dan», creo que pensé yoł. 
Como forma de resistencia a las dinámicas de exclusión de la urbe, que conducen muchas veces a las mujeres Embera a ejercer la mendicidad en las calles, Jimbûso Wera optó por trabajar las artesanías, fundamentalmente el tejido en mostacilla, esperando con esto lograr una estabilidad económica que le permitiera mantener a su familia. En este oficio, encontró una opción digna de trabajo, que le asignaba un rol diferente dentro de la comunidad, una ruptura/apertura experiencial que la llevaba a ser educadora de quienes por diferentes razones no sabían tejer, e incluso cambiar el rol dentro de su familia y pasar a ser la principal proveedora, lo que implica la inversión de los roles de género. Frente a su papel en la comunidad, Jimbûso Wera relata cómo criticaba la opción de las otras personas de ejercer la mendicidad:

Yo le decía a la gente «no vayan a pedir monedas, miren que yo no pido desde que yo llegué, siendo que yo llegué [después de] ustedes ${ }^{\star}{ }^{\prime}$. Y yo cuando llegué aquí [habían] personas que eran más adelantes, que son desplazados llevaban de tres años $\uparrow$, de cuatro años. Entonces ellos si como no rebuscaban ni nada $\downarrow$ Entonces yo les dije, jah no!

Progresivamente y de manera perseverante, el trabajo artesanal de Jimbûso Wera fue reconocido por otros como una buena opción, lo que posibilitó que ella enseñara a otros Embera el tejido en mostacilla, comenzando a ser vista como líder y educadora de la comunidad. Incluso, de forma paradójica, su conocimiento del tejido y su relevancia para la estabilidad económica en la ciudad propició una inversión muy particular de los roles educativos, pues Jimbûso Wera enseñó a sus padres el oficio del tejido Embera. En este marco, la ciudad no sólo era escenario de transmisión de conocimientos y prácticas tradicionales, sino que, paradójicamente, la hija pasaba a ser educadora de sus padres en una práctica cultural tradicional, la cual había aprendido de su abuela paterna:

Sí a él [a mi papá] yo le enseñé $\uparrow$, a mi mamá también, mi mamá por parte de chaquiras no sabía nada. Mi mamá no sabía tejer $\uparrow$, solo sabía hacer de bejuco no más, pero de chaquira no. [A mí] Eso sí [me] enseñó mi abuelita [...]. Ella como sabía hacer muchas cosas $\downarrow$ [.. . ]cántaros de::, de barro, cerámicas, tejidos de bejucos, también me enseñaron ellos, me enseñó a parte de eso tejido de chaquira. [...] A mi mamá también me tocó enseñarle [...] ellos hacían cosas muy feas, pero:: [...] Mi papá también hacía colores sin combinar $\uparrow$, que no me gustaba. Yo le dije a mi papá que tenía que combinar los colores que son de [...] pálido hacia vivo. Y se quedaban perfectamente bonitos $\uparrow$. $Y$ ya saben combinar bien sus colores, saben vender, mi mamá sabe vender en la calle también^.

En particular, las dinámicas que ha suscitado el tejido en la ciudad merecen un análisis particular que sobrepasa los alcances de este texto; sin embargo, considero que esta práctica puede ser leída como una forma de resistencia cultural en la ciudad, en la cual la tradición sobrevive a los constreñimientos de lo urbano, pero no se encuentra exenta de constreñimientos (por ejemplo en sus diseños y formas de comercialización) que pueden reducir su valor simbólico, ubicando en segundo plano o incluso olvidando los significados tradicionales, y reduciendo el tejido a una práctica importante por su valor económico. A nivel de las dinámicas de género y como otros estudios lo exponen (Cabrera, et al., 2009; Gaitán, 2010), el tejido en Bogotá ha conllevado un cambio 
importante en los roles tradicionales, puesto que las mujeres a través de la actividad artesanal han comenzado a ser las proveedoras del hogar, saliendo a trabajar en las calles, mientras que la mayoría de los hombres se quedan en el hogar cuidando a los niños y tejiendo algunos de los artículos para vender. Esta transformación, a nivel general, modifica los espacios sociales tradicionalmente asignados para hombres y mujeres en el territorio, puesto que lo femenino se ubica en la esfera pública, mientras que lo masculino pasa a situarse en el ámbito de lo privado. Como lo sostiene Alonso (2008) sobre las familias indígenas en Bogotá, la vinculación de la mujer al trabajo remunerado juega un importante papel a la hora de los cambios culturales en los roles de género, lo cual es reflejado biográficamente en los relatos de Jimbûso Wera.

En estos complejos juegos de poder, resistencia y cambio cultural, un hito fundamental en la vida de Jimbûso Wera por la relevancia, fuerza y reiteración con la que se presenta en su relato personal, fue su proceso de separación, que merece un alto en el camino ante el cisma y la escisión biográfica que produce. Como se mencionaba con anterioridad, desde el territorio Jimbûso Wera era fuertemente maltratada por su esposo, por lo cual había intentado en varias ocasiones separarse de él, y aunque vivió sola un tiempo donde sus padres en Mistrató, volvió con él y juntos salieron del territorio hacia Bogotá. Las razones por las cuales sus intentos de separación en el territorio no tuvieron éxito están configuradas no sólo por la tradicional asimetría de género entre los Embera sino por su fusión y argumentación desde nociones católico-occidentales, por lo cual las restricciones culturales hacia las mujeres se encontraban hibridadas con valores católicos como el "matrimonio hasta la muerte":
Yo allá [en territorio] no podía separarme. [...] Porque los cabildos no dejan separar, y me dijeron que yo no podía separar, como yo me casé por la iglesia me dijeron que a mí me iban a castigar. Aunque mi esposo esté maltratando como es la culturał que yo tenía que aguantar hasta la muerte, no podía y yo me aguantaba por eso [...] Allá en territorio no dejan separarł. Si usted va a separar ellos le castigan en territorio a la mujer por seis meses tienen que limpiar, hacerse siembra de todos $\uparrow$ y no le pagan a uno. Los gobernadores le llevan, le dan machete y ellos tienen que estar mirando la obra de uno.

Dicha argumentación sobre la justificación del maltrato femenino y la imposibilidad de separarse combina las costumbres indígenas con los preceptos católico-occidentales y es un ejemplo de lo que Santos (2009) denomina hibridaciones reaccionarias, en las cuales las mezclas culturales mantienen esquemas de dominación y asimetría, por contraposición a las hibridaciones emancipatorias que dan voz al subalterno a partir de la fusión cultural. Fue tan sólo después de su llegada a Bogotá, cuando Jimbûso Wera pudo finalmente separarse:

Yo me separé fue cuando me vine acá a Bogotá desplazándome $\downarrow$. Y aquí también lo mismo siguió [...] con ese maltrato [...] cuando mis papás llegaron yo ya había echado a ese, a ese señor $\uparrow$. Ya estaba sola, porque desde esa época yo ya me defendía sola.

De esta manera, la ciudad se perfiló en este caso como escenario de empoderamiento y agencia para que Jimbûso Wera tomara esta decisión, puesto que las restricciones de las autoridades tradicionales no tenían presencia en la urbe. Esta compleja opción 
implicaba, sin embargo, una ruptura con los cánones culturales, un acto de resistencia a través de la construcción de un sendero alternativo a la tradición y la re-significación de su existencia, no en función de la vida en pareja sino a partir de trayectorias vitales más autónomas y empoderadas. Dos aspectos adicionales jugaron un importante papel en este proceso de separación. En primer lugar, Jimbûso Wera había comenzado a trabajar como gestora de salud en un hospital de Bogotá, y existía la posibilidad de perder este trabajo ante las prohibiciones de su esposo. En segundo lugar, el apoyo de la familia y en particular de su propio padre fueron fundamentales al consentir su decisión de terminar la vida de pareja con su esposo:

Casi me sacaron del trabajo que porque mi esposo se volvió celoso por esol. Y una vez «Jimbûso Wera así no podemos, usted tiene que, tenía que trabajar pero su esposo no la deja ¿qué vamos a hacer? $\downarrow$ ». Me dijeron «si usted renuncia de su esposo con mucho gusto acá [habría] trabajo otra vez, y si no pues ya tocará dejar el trabajo \». [...] Después más bien yo voy a vivir sola, pensé. Hablé con mi papá le dije «mire papá me está pasando esto", me dijo «ah bueno eso es su decisión». [...] «La decisión es de usted, y además yo también he visto^ que como que no quiere a los niños tampocol, entonces es mejor que separen». Y ya me quedé sola desde esa época y me quedé trabajando con salud.

Ahora Jimbûso Wera asumía un rol sui generis para la comunidad Embera: madre cabeza de familia. Esta situación no es, sin embargo, completamente inédita, pues como lo sostiene Alonso en su estudio sobre las familias indígenas en Bogotá, "muchas mujeres indígenas [...] sobre todo en las grandes ciudades, conquistan espacios de liderazgo y au- tonomía que no les era permitidos en sus comunidades ancestrales y, en consecuencia, asumen la conducción de la familia" (2008, p.232). Esta situación impulsó igualmente su papel como lideresa comunitaria, pues hace más de tres años, Jimbûso Wera comenzó a trabajar como gestora de salud de la comunidad Embera para el Hospital de Centro Oriente, ubicado en el centro de Bogotá. Ante las dificultades institucionales para la atención en salud para las familias Embera en condición de desplazamiento, el hospital le propuso a Jimbûso Wera trabajar con ellos facilitando la traducción y acompañando a la comunidad en los servicios médicos ofrecidos por las instituciones distritales. En la ciudad, el dominio del español le ha facilitado ser un puente institucional, cultural y social entre la comunidad y el mundo urbano-occidental, ubicándose en la frontera y moviéndose constantemente entre lo propio y lo ajeno. De igual forma, este trabajo ha implicado la exploración de nuevos conocimientos, el tránsito por nuevos senderos que la han llevado a hablar desde otras voces, apropiándose de otros discursos desde los cuales trabaja ahora no sólo con la comunidad Embera sino con otras comunidades indígenas de Bogotá: "Lo que nosotros $\uparrow$ hacemos es sensibilizar a la gente, a la comunidad indígena, como ellos no saben acerca de esas enfermedades, entonces nosotros hacemos sensibilización ł".

En continuidad con lo mencionado, el acercamiento a la medicina occidental que ha significado esta experiencia para Jimbûso Wera, ha movilizado su lectura sobre la salud y el jaibanismo como creencia tradicional con su respectivo sistema de comprensión de la salud y la enfermedad. De manera reflexiva, Jimbûso Wera cree en la necesidad de complementar y mezclar la medicina occidental y la tradicional, inclinándose -sin argumentarlo 


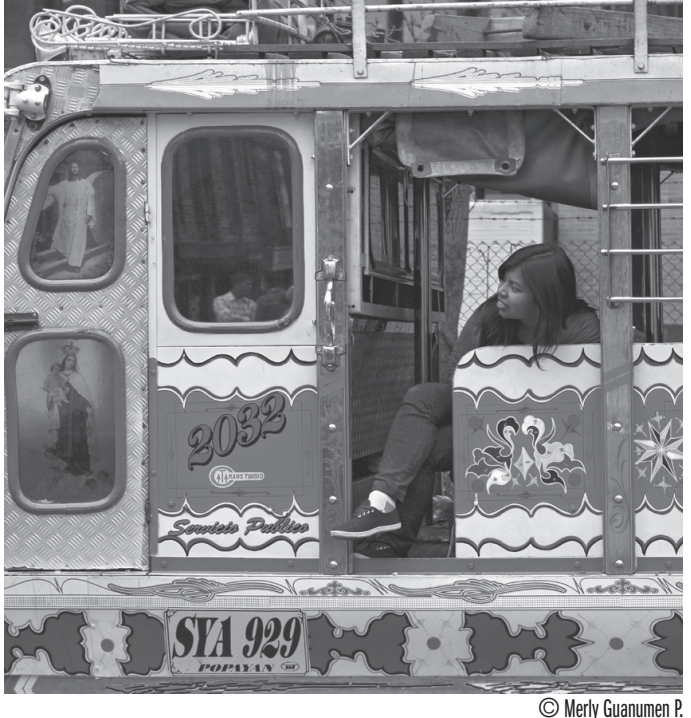

de tal forma- por un diálogo de saberes y un proceso intercultural. Esta lectura de la relación entre el jaibanismo y la medicina occidental puede conllevar a hibridaciones emancipatorias (Santos, 2009) en la ciudad, puesto que no subalternizan los conocimientos y prácticas tradicionales sino que las sitúan de manera legítima en relación con las lógicas occidentales.

Sí entonces yo he tratado [con jaibaná], yo creo en eso, pero no creo mucho, porque igual como nosotros ya creemos las dos cosas, ehh, de enfermedad occidental $\uparrow$ y enfermedad de nosotros, entonces yo siempre creo en las dos cosas, porque como ahora tengo conocimiento que en el tema de la enfermedad tuberculosis por ejemplo eso no se va, no se cura con nuestro jaibaná $\uparrow$, eso tiene que ser directamente tratado con los medicamentos occidentales, porque esa enfermedad es muy, o sea, es:: dicen que es grave si no se detecta a tiempo $\downarrow$ [...]. Entonces por esa razón nosotros siempre en la comunidad venimos explicando que tiene que saber pues muchas cosas $\downarrow$ de medicinas ancestrales, pero tiene que combinar las dos cosas, que creer en tanto occidental como los médicos ancestrales $\downarrow$.

El último de los momentos o hitos identificados en los relatos biográficos de Jimbûso Wera, se encuentra ligado a los escenarios educativos en la ciudad, en particular algunos espacios de educación formal en donde han compartido con kapunías ${ }^{7}$, y se han acercado de manera más contundente a los conocimientos occidentales. Particularmente, Jimbûso Wera fue una de las primeras mujeres Embera en participar de estos espacios, al entrar a validar el bachillerato en el Colegio La Candelaria. Según relata, esta experiencia no estuvo exenta de dificultades:

yo mejor dicho he estudiado mucho, en muchos colegios ((riendo)). Mire, ahí me puse a estudiar pero como esos días yo como tenía que trabajar $\uparrow$, a veces me tocaba venir en la noche a acompañar al médico a los pacientes, por eso también me perdí el semestre $\downarrow$.

En los relatos de Jimbûso Wera no se identifican dinámicas de exclusión directa por parte de compañeros o profesores; sin embargo, estos conocimientos escolares marcan sin duda esquemas de pensamiento distintos a los tradicionales, lo que se evidencia con relativa fuerza frente al aprendizaje de las ciencias naturales, como un sistema de pensamiento que entiende al mundo natural de una forma distinta en comparación a la aprendida en el territorio desde la medicina tradicional. Jimbûso Wera, motivada por el trabajo como gestora de salud, comenzó a estudiar enfermería en un instituto técnico y de manera paralela continúa validando el bachillerato en el Colegio Camilo Torres, donde a diferencia de las

7 Es la palabra en Embera que designa al no indígena. 
dinámicas de colonialidad vividas en el internado que excluían cultural y epistemológicamente lo no occidental (Castro-Gómez, 2005), ha establecido relaciones no discriminatorias y de cooperación con compañeros no indígenas. Sin embargo, dichas relaciones no pueden considerarse como intercambios recíprocos y horizontales de conocimientos, pues queda la duda de qué elementos tradicionales Embera los estudiantes kapunías han aprendido o están dispuestos a conocer:

Yo primero estudié solo yo, como en intermedio de los blancos, yo solita estabał. Ehh, pues si chévere porque hay otros que les gusta no, 0 sea no se discrimina a uno que porque es indígena. [...] jAy no!, allá fue muy bueno::., todos los alumnos. Me ayudaban y todo, y me sacaron adelante, ellos me enseñaban, pues era muy difícil aprender matemáticas. Pero una compañera me ayudó también y ahí $[$ [...] una compañera blanca.

En su conjunto, esta serie de experiencias educativas formales en la ciudad, han transformado la vida de Jimbûso Wera, no solo en sus actividades cotidianas, sino en el rol que juega en la comunidad y en las formas en las cuales se ve a sí misma. La fuerza de su agencia la han llevado a plantear senderos alternativos a los vividos por los demás miembros de la comunidad, evidenciando un tránsito de lo indígena-tradicional hacia lo urbano-occidental con múltiples aristas, con fluctuaciones emocionales, sociales y culturales, que no permiten una narración estática sobre sí misma, sino un vaivén continuo que marca a la vez apertura y nostalgia por lo tradicional. Como ejemplo de la vitalidad que acompaña la voz de Jimbûso Wera en su relato, retomo sus intenciones de continuar estudiando, pues son motivaciones viscerales que sobrepasan los intereses económicos o de ascenso social:

[Yo estudio] para tener conocimiento, pues para tener conocimiento $\downarrow$ y aparte de eso para poder aprender cosas y para seguir estudiando en algo $\downarrow$ [...] yo no estoy estudiando que porque voy a ganar más dinero, voy a ganar más plata, noł. Sino que mi decisión es tener conocimiento en el estudio^ [...]. Eso es lo que he enseñado a mis hijos $\downarrow$, que uno no debe tener ese pensamiento malo, que yo voy a ganar mucho dinero, por eso voy a estudiar $\downarrow$. Yo siempre le digo a mis hijos «uno estudia es para tener conocimiento y aprender las cosas».

\section{A modo de (in)conclusión}

La historia de Jimbûso Wera es, en gran parte, la historia de las mujeres y de la comunidad Embera, sus experiencias son microcosmos de las complejas relaciones entre violencias, poderes y resistencias de este pueblo indígena. Su histórica resistencia a los españoles durante la conquista, que los llevaba a dispersarse una vez culminados los enfrentamientos (Losonczy, 2006), la invasión de sus territorios, primero por parte colonos republicanos y posteriormente de campesinos igualmente desterrados, el consecuente proceso de evangelización liderado por misiones católicas (Vasco, 1975), las vulneraciones producto del conflicto armado y la violencia socio-política que azota al país (Villa \& Houghton, 2005), y finalmente el conjunto de marginaciones y exclusiones que conforman su desplazamiento en la ciudad, hacen parte de la violencia estructural sobre los pueblos indígenas reflejada en la historia de la comunidad Embera y en la historia personal de Jimbûso Wera. Diametralmente constituida por una lógica etno-céntrica y colonial, las variables 
formas en las cuales se manifiesta la violencia contra los indígenas y en particular contra las mujeres indígenas, obedece al ejercicio de poder que jerarquiza, subalterniza y excluye. Como lo sostiene Restrepo (citado en Ulloa, 2007), es evidente en el caso de las mujeres indígenas el impacto de todas las formas de violencia que se ejercen tanto por actores ilegales (grupos armados, por ejemplo) como por el mismo Estado, quien por omisión o acción vulnera sus derechos sistemáticamente.

A pesar de este turbio panorama, mujeres como Jimbûso Wera y otras mujeres indígenas de forma altamente disímiles "han desafiado las tradiciones [y] han incursionado en múltiples espacios de la ciudad" (Méndez, 2007, p.41), han resistido mediante estrategias, acciones y experiencias -no exentas de paradojas- las múltiples exclusiones dentro y fuera de sus comunidades. Estas dinámicas permiten entrever cómo las resistencias se configuran más allá de lo reactivo, caracterizándose particularmente por su carácter creativo y productivo, en esa estrecha relación entre vida, resistencia y creación (Giraldo, 2006). Como lo sostiene Foucault, la resistencia es en comparación a su contraparte, el poder, "tan inventiva, tan móvil, tan productiva como él. Es preciso que como el poder se organice, se coagule y se cimiente. Que vaya de abajo arriba, como él, y se distribuya estratégicamente" (2000, p.162). Las experiencias vitales de Jimbûso Wera encarnan en sí mismo tales fluctuaciones entre poderes y resistencias, manifestando confluencias, desencuentros y paradojas entre lo indígenatradicional y lo urbano-occidental. Por supuesto, dichas formas femeninas e indígenas de resistencia no están al margen de conflictos, en particular los relacionados con sus procesos identitarios, pues "cualquier identidad no es una entidad monolítica de puro poder o de pura resistencia" (Restrepo, 2012, p.139).
En un tránsito constante entre lo propio y lo ajeno, entre lo indígena y lo urbano-occidental, las formas de resistencia contempladas a lo largo de la vida de Jimbûso Wera, son reflejo de ese sujeto en fuga propio de la resistencia (Giraldo, 2006), en el cual la transgresión parece ser la norma, en ese continuo surgimiento de posiciones de enunciación, de desestabilización de axiomas y categorías en el que se construyen las mujeres indígenas en la ciudad (Maeso, 2008). En esa lógica de frontera entre lo tradicional y lo occidental, poderes, resistencias y vidas se ponen en juego en un vaivén incesante que nunca cierra las identidades en juego pero que a la vez no las abre de par en par. Esta movilidad fronteriza se hace evidente en las siguientes narraciones, la primera, el testimonio de una mujer Uitoto en Bogotá recopilado por Alonso: "En la familia la mujer debe llevar dos roles, cuando voy a mi comunidad tengo que abrir chagras, ando descalza, y cuando estoy acá soy una mujer de zapatos altos y me maquillo" (2008, p.235). La segunda narración, análoga de algún modo a la anterior, es la referida por Jimbûso Wera, quien argumenta la centralidad de la lengua y el vestido como núcleo de identificación y resistencia como mujer indígena; sin embargo, refleja al mismo tiempo la permeabilidad y las fugas del mismo: "no pueden ehh::, de dejar hablar Embera, porque si nosotros dejamos eso totalmente nos puede perder la culturał. Aunque somos indígenas nadie se va a reconocer que somos indígenas". Con relación al vestido, afirma: "Entonces yo aquí así por el momento tengo $\downarrow$ un vestido occidental, pero cuando yo llego a mi territorio, si me dan casa yo me quito esta ropa $\uparrow$, y prefiero vestir mi vestido, porque eso sí no lo dejaría".

En definitiva, la complejidad de estas dinámicas nos permite una última conclusión; su movilidad y paradójica configuración bajo nin- 
guna circunstancia autorizan identificar "esencias" o "naturalezas" de las resistencias, las relaciones de poder y los modos de violencia que viven las mujeres indígenas en condición de desplazamiento en las ciudades. Queda por supuesto el gran reto de abandonar la visión unidireccional dominante-dominado, y considerar en toda su complejidad las relaciones humanas en sus dinamismos y contradicciones, pero en particular, en sus interfaces entre poderes y resistencias (Foucault, 1992). Por último, considero fundamental reconocer la tenacidad de mujeres como Jimbûso Wera, quien lejos de estar absuelta de conflictos, ambivalencias o paradojas, encarna en su historia personal la fortaleza, la resistencia y la vitalidad de las mujeres indígenas de Colombia, quienes cotidianamente luchan en contraposición a las múltiples violencias que las aquejan.

\section{Referencias bibliográficas}

- Alonso, M. (2008). Familias indígenas en Bogotá. En M. Gutierrez (Ed.), Las familias en Bogotá. Realidades y diversidades (pp.207-255). Bogotá, Colombia: Pontificia Universidad Javeriana.

- Archila, M. (1998). Fuentes orales e historia obrera. En T. Lulle; P. Vargas \& L. Zamudio (coord.), Los usos de la historia de vida en las ciencias sociales Tomo I (pp.281 - 296). Barcelona, España: Anthropos.

- Bourdieu, P. \& Wacquant, L. (2005). Una invitación a la sociología reflexiva. Buenos Aires: Siglo XXI Editores.

- Briones, C. (2010). "Nuestra lucha recién comienza": Experiencias de pertenencia y de formaciones mapuches del yo. En M. de la Cadena \& O. Starn (Ed.), Indigeneidades contemporáneas: Cultura, política y globalización (pp.115 - 137). Lima: Instituto de Estudios Peruanos.

- Cabrera, F.; Barbero, C.; López, F.; Castillo, A.; Caicedo, Y.; Bravo, D. \& Alzate, D. (2009). Identificación y caracterización socioeconómica, cultural y nutricional del observatorio de la situación de los niños, niñas y de las familias Embera en Bogotá. Bogotá, Colombia: ICBF-Vía Plural.

- Castro-Gómez, S. (2005). La postcolonialidad explicada a los niños. Popayán, Colombia: Universidad del Cauca.

- De la Cadena, M. (1991). "Las mujeres son más indias": Etnicidad y género en una comunidad del Cusco. Revista Andina, 9(1), 7-45.

- Foucault, M. (1992). Microfísica del Poder. Madrid, España: Ediciones La Piqueta.

- Foucault, M. (2000). Un diálogo sobre el poder y otras conversaciones. Madrid, España: Alianza Editorial.

- Gaitán, S. (2010). Identidades Embera en Bogotá: Un presente nebuloso entre las memorias de un pasado y la esperanza incierta de un futuro. (Tesis de pregrado). Universidad Nacional de Colombia, Bogotá, Colombia.

- Giraldo, R. (2006). Poder y resistencia en Michel Foucault. Revista Tabula Rasa, (4), 103-122.

- Losonczy, A-M. (2006) Viaje y violencia: la paradoja chamánica emberá. Bogotá, Colombia: Universidad Externado de Colombia.

- Maeso, S. (2008). Relaciones de poder e inscripciones identitarias: Mujeres y diferencia cultural indígena en contextos latinoamericanos. Revista Ex aequo, (17), 53-70.

- Mejía, W. (2007). Presencia Embera en el Área Metropolitana Centro Occidente. Pereira, Colombia: Fundación Cultural Germinando.

- Méndez, G. (2007). Nuevos escenarios de participación: experiencias de mujeres indígenas en México y Colombia. En L. Donato; E. Escobar; P. Escobar; Pazmiño A. \& A. Ulloa (coord.), Mujeres indígenas, territorialidad y biodiversidad en el contexto latinoamericano (pp.35-46). Bogotá, Colombia: Universidad Nacional de Colombia.

- Merino, M. \& Tileagâ, C. (2011). La construcción de identidad de minorías étnicas: un enfoque discursivo psicológico a la autodefinición étnica en acción. Discurso \& Sociedad, 5(3), 569-594.

- Ministerio de Cultura de Colombia (2010a). Caracterización del Pueblo Emberá Katío. Recuperado de http://bit.ly/1uVOHuN

- Ministerio de Cultura de Colombia (2010b). Caracterización del Pueblo Emberá Chamí. Recuperado de http://bit.ly/1ucC7Tz

- Oehmichen, C (1999). La relación etnia-género en la migración femenina rural-urbana: Mazahuas en la ciudad de México. Revista Iztapalapa, (45), 107-132.

- Peñaranda, R. (2012). Nuestra vida ha sido nuestra lucha. Resistencia y memoria en el Cauca indígena. Bogotá, Colombia: Centro de Memoria Histórica.

- Potter, J. (1998). La representación de la realidad. Discurso, retórica y construcción social. Barcelona, España: Paidós.

- Restrepo, E. (2012). Intervenciones en teoría cultural. Popayán, Colombia: Universidad del Cauca.

- Sabogal, J. (2014). Experiencias educativas y construcciones del Yo en comunidades Embera: una mirada biográfica (Tesis de pregrado). Universidad Nacional de Colombia, Bogotá, Colombia.

- Santos, B. (2009). Una epistemología del sur: La reinvención del conocimiento y la emancipación social. México: Siglo XXI-CLACSO.

- Ulloa, A. (1992). Los Embera. En A. Chaves (Coord.), Geografía Humana de Colombia. Región del Pacífico. Tomo IX. Bogotá, Colombia: Instituto Colombiano de Cultura Hispánica. Recuperado de http://bit.ly/1pRKi3D

- Ulloa, A. (2007). Introducción: Mujeres indígenas: dilemas de género y etnicidad en los escenarios latinoamericanos. En L. Donato; E. Escobar; P. Escobar; Pazmiño A. \& A. Ulloa (coord.), Mujeres indígenas, territorialidad y biodiversidad en el contexto latinoamericano (pp.17-34). Bogotá, Colombia: Universidad Nacional de Colombia.

- Vasco, L-G. (1975). Los Chamí. La situación del indígena en Colombia. Bogotá, Colombia: Editorial Margen Izquierdo, Colección Populibros, № 1.

- Vasco, L-G. (1990). Jaibaná Embera y Chamanismo. En Cultura Embera. Memorias del Simposio sobre Cultura Embera. Medellín, Colombia: Organización Indígena de Antioquia.

- Vasco, L-G. (2002). Entre selva y páramo: viviendo y pensando la lucha india. Bogotá, Colombia: Instituto Colombiano de Antropología e Historia.

- Villa, W. \& Houghton, J. (2005). Violencia política contra los pueblos indígenas en Colombia 1974-2004. Bogotá, Colombia: Centro de Cooperación al Indígena -CECOIN-y Organización Indígena de Antioquia -OIA-.

- Yanes, P. (2004). Urbanización de los pueblos indígenas y etnización de las Ciudades. Hacia una agenda de derechos y políticas públicas. En P. Yanes; V. Molina \& O. González (Coord.), Ciudad, Pueblos Indígenas y Etnicidad (pp.191-223). México: Universidad Autónoma de la Ciudad de México. 